(4) While each of the three preceding books is good in its way and within its limitations-which are those of examination syllabuses-and may be accepted as a reliable medium of instruction, they lack enthusiasm. They work methodically and conscientiously through the allotted task, but will not evoke in the student a wider interest in the subject. In sharp contrast, Prof. Anderson takes up his subject with an obvious sense of its importance and of its absorbing interest. While writing for students of science, agriculture, engineering and other branches of applied physics, he urges its cultural value to all. This is not to suggest that his treatment departs from the logical and recognized course, but rather to indicate the spirit in which the instruction is given and the easy introduction of practical topics to illustrate and reinforce it. For example, after dealing with fluids in motion and reduction of pressure with increase of velocity, he leads up to a discussion on carburettors, ship collisions, pitot tubes, the rotor ship, venturi meters, steam injectors, organ pipes and several other examples. With such a book the student will gain greatly in his mental outlook.

(5) The last of these volumes is in a different category. It contains a symposium of essays on the applications of physics in industry by different American authorities in the glass, metal, oil and other industries. Though by no means equal in quality, they all, with perhaps one exception, reach a high standard of interest and of technical value. It is a book which should be read not only by every scientific worker and engineer but also very widely by the general public, to whom it would convey the vast importance of physical science in modern life.

\title{
Analysis of Animal Foods
}

The Structure and Composition of Foods

By Dr. Andrew L. Winton and Dr. Kate Barber Winton. Vol. 3 : Milk (including Human), Butter, Cheese, Ice Cream, Eggs, Meat, Meat Extracts, Gelatin, Animal Fats, Poultry, Fish, Shellfish. Pp. $\mathrm{xxv}+524$. (New York: John Wiley and Sons, Inc. ; London: Chapman and Hall, Ltd., 1937.) 40s. net.

$W^{\text {HEN the two preceding volumes of "Winton }}$ and Winton" were issued, we believed, as did the authors, that the third volume would complete their work. Apparently, however, they had under-estimated the range of products needing survey, and they have now had once more to postpone the end of their labours. The publishers state that volume 4 , in preparation, will cover the remaining foodstuffs-sugar, syrup, tea, coffee, cocoa, spices, extracts, etc. Volume 3, however, will be for many the climax of this important and useful work, for the analysis and control of animal foods offer, broadly speaking, greater diffculties to the technologist than do even some of the knottier problems in the control of cereals and seed oils.

Books of this type must always suffer from one inherent disadvantage. In this volume, for example, it must have been very difficult to decide exactly how much space to allot to milk and milk products ; actually they receive 209 pages. Eggs (in Part 2) occupy a further 50, miscellaneous animal products some 30 , meat and meat products just under 140, fish and fish products a further 50 .
The remaining pages are devoted to batrachians and reptiles and to an index.

It is clear that 200 pages about milk and milk products constitute, in effect, a monograph on the composition and analysis of dairy products matters that have been very adequately treated in a number of specialized books. Is there really any call for its treatment at such length here? If, on the other hand, it had been decided that much shorter treatment would be satisfactory, the authors would immediately have laid themselves open to the charge of inadequacy.

We must admit that this is a problem that we are glad not to need to face. The authors, however, have had to face it and, granted the premises that decided their editorial policy, have done so with considerable success. It is, of course, impossible to check anything but random samples of the facts summarized in such a book. References appear to be brought up to 1936, and include such recent information as the Levene formulæ for yeast nucleic acid and thymus nucleic acid, and for inosinic acid.

The book is unfortunately not free from gross printer's errors. For example, "Greatine" on page 275. Also, much of the information collected by the authors about the effect of cooking on meat (and other foods) has now been rendered obsolete by the studies of McCance and Widdowson. Nevertheless, the book will remain permanently useful, not only as a record of facts, but also as a record of the kind of facts that have been recorded.

A. L. B. 